\title{
INOCULAÇÃO DE SUBSTRATO COLONIZADO COMO ALTERNATIVA AOS PEQUENOS PRODUTORES DE COGUMELOS COMESTÍVEIS NO BRASIL
}

\section{ALTERNATIVE INNOCULATION OF SUBSTRATE FOR SMALL-SCALE EDIBLE MUSHROOM PRODUCERS IN BRAZIL}

\author{
Emerson Tokuda Martos ${ }^{1}$, Karina Heck ${ }^{2}$
}

1,2 Cogumelos do Japinha, Bom Sucesso, Minas Gerais, Brasil - etmartos@yahoo.com.br \& kheck_bio@hotmail.com

\section{RESUMO}

\begin{abstract}
A produção de cogumelos comestíveis do gênero Pleurotus sp., popularmente conhecidos como shimeji, é amplamente difundida no Brasil, em virtude da facilidade de cultivo e de suas propriedades nutricionais. Como decompositor primário, promovem a ciclagem de nutrientes oriundos de resíduos diversos. Pequenos produtores devem se adequar a requisitos para alcançar boa produtividade, em especial, substrato de boa qualidade e acessível, e fonte de inóculo vigoroso que supra as demandas. O objetivo dessa pesquisa é avaliar a viabilidade na produção de cogumelos a partir de composto inoculado com alíquota de outro composto previamente colonizado por micélio, comparada com o método tradicional de inoculação ("spawn"). Os dois sistemas de inoculação foram comparados quanto à velocidade de colonização do composto e produtividade, em gramas de cogumelo colhido. Os resultados não mostraram diferença estatística entre os dois experimentos, apontando alternativa sustentável e viável na substituição de inóculo aos pequenos produtores, especialmente na indisponibilidade do inóculo comercial. É, portanto, uma alternativa que viabiliza o ciclo agroecológico na propriedade rural, tornando, inclusive, a produção de cogumelos comestíveis mais rentável e menos onerosa ao pequeno produtor.
\end{abstract}

PALAVRAS-CHAVE: Compostagem, Crescimento micelial, Inóculo alternativo, Pleurotus ostreatus

\section{ABSTRACT}

The production of edible mushrooms of the genus Pleurotus sp., commonly known as shimeji, is widespread in Brazil, due to the ease of cultivation and its nutritional properties. As a primary decomposer, it promotes the cycling of nutrients from different agricultural wastes. Small-scale edible mushroom producers must adapt to requirements to achieve good productivity, in particular good quality and accessible substrate and source of vigorous spawn. The aim of this study was to evaluate the viability in the production of mushrooms from a compost inoculated with an aliquot of another compost previously colonized by mycelium, compared with the traditional method of inoculaton - spawn. It was compared in terms of colonization speed and productivity. The results showed there was no statistical difference between the two experiments, suggesting a sustainable and viable alternative in the inoculum replacement for small-scale edible mushroom producers, especially in the absence of the commercial spawn. Therefore, this is an alternative that enables the agroecological cycle in rural zones, performing even the production of edible mushrooms more profitable and less costly for small-scale producers.

KEYWORDS: Alternative spawn, Composting, Mycelial growth, Pleurotus ostreatus 


\section{INTRODUÇÃO}

Cogumelos comestíveis são fontes de proteínas, micronutrientes e moléculas bioativas benéficas à saúde, especialmente no combate aos efeitos do estresse oxidativo (SÁNCHEZ, 2010; SILVA et al., 2013; OGIDI et al., 2016). Além de utilizados na alimentação e em outras aplicações biotecnológicas, participam de processos de decomposição de matéria orgânica, promovendo a ciclagem de nutrientes na natureza e garantindo seu retorno ao solo. Assim, os resíduos gerados a partir do esgotamento do composto de produção de cogumelos podem ser utilizados como condicionador de solos e em culturas de hortaliças (RIBAS et al., 2009; MARQUES et al., 2014; LOPES et al., 2015).

Seu consumo no Brasil tem sido encorajado nas últimas décadas e, por conseguinte, a expansão do cultivo tem sido expressiva. Os três gêneros de Basidiomyceto mais cultivados são Agaricus sp. L. ex. Fr. (Champignon, Porto Belo e Cogumelo do Sol), Pleurotus sp. (Jacq. Fr.) Kumm (Shimeji) e Lentinula sp. (Berk.) Pegler (Shiitake). De acordo com a Associação Nacional dos Produtores de Cogumelos, o gênero Pleurotus sp. corresponde ao segundo mais cultivado, correspondendo à produção de mais de $16 \%$ (2000 tons/ano) do total de fungos comestíveis. São cultivados em uma ampla diversidade de substratos (SALES-CAMPOS et al., 2011b; CHITAMBA et al., 2012; GIRMAY et al., 2016), e respondem às oscilações dos fatores de crescimento, como temperatura, composição química do substrato (nutrientes, relação carbono/nitrogênio e recalcitrância), teores de umidade, dióxido de carbono e oxigênio (ZADRAZIL, 1975; OBODAl et al., 2003; SALES-CAMPOS et al., 2011a; MANTOVANI et al., 2012).

O sucesso da produtividade, portanto, deve-se às condições ótimas e controladas de cultivo, e a seleção de substratos de qualidade e de fácil aquisição permite a redução de custos de cultivo. Por consequência, a demanda crescente e a expansão do mercado brasileiro induzem a busca constante por aprimoramento das técnicas produtivas (DIAS, 2010; CARVALHO et al., 2012; SIQUEIRA et al., 2012). Sendo assim, a ampliação do cultivo em pequena escala também é definida pela aquisição do inoculante ("spawn") e/ou substrato de qualidade (Zharare et al., 2010). O planejamento da produção, desde a seleção do substrato, aquisição do inóculo, processo de colonização do composto (crescimento micelial) e colheita, definem a produtividade e a perspectiva de expansão da área de cultivo (SIQUEIRA et al., 2012; VIEIRA e ANDRADE, 2016). Por consequência, a interrupção no fornecimento de inóculo e/ou fontes de contaminação acarretam prejuízo produtivo e financeiro, pois causa atraso no cronograma de execução, muitas das vezes necessitando o descarte do substrato ainda na fase de inoculação.

A inoculação a partir de um composto previamente colonizado pode ser uma alternativa viável para evitar essas perdas, possibilitando maior autonomia produtiva, visto que garante fonte de inóculo de emergência em situação de falta de "Spawn" pelo mercado fornecedor. Trata-se da remoção de uma alíquota de composto sadio e densamente colonizado, transferido para um composto recém preparado, induzindo a colonização do material. Assim, o objetivo proposto por este trabalho é avaliar o uso do composto previamente colonizado como alternativa aos inoculantes tradicionais no cultivo de shimeji (Pleurotus ostreatus) em um sistema de compostagem.

\section{MATERIAL E MÉTODOS}

\section{Área experimental}

O experimento foi conduzido na cidade de Bom Sucesso, região sudeste de Minas Gerais, Brasil, entre Junho e Dezembro de 2018. A temperatura média da região varia de $15 \circ \mathrm{C}-23.5 \circ \mathrm{C}$, com amplitude térmica média de 8.5ำ e precipitação média variando de 19 - 281 $\mathrm{mm}$ para o período (Climatempo).

\section{Estufa de cultivo}

A estufa de cultivo corresponde a uma área de $250 \mathrm{~m}^{2}$ cobertos com lona de vinil dupla face branca/preta, com a face branca voltada para a área externa, e tela de sombreamento $50 \%$ nas extremidades superiores, para promover a troca gasosa. A umidade atmosférica é mantida à saturação próxima a $80 \%$, por sistema de nebulização.

\section{Substrato do composto e inoculante comercial}

Foi utilizado bagaço de cana-de-açúcar (Saccharum officinarum L.) triturado a $5 \mathrm{~cm}$ de tamanho, feno de braquiária [Brachiaria decumbens (Trin.) Griseb.] e palha de feijão (Phaseolus vulgaris L.) triturada a $5 \mathrm{~cm}$ de tamanho, utilizados em uma proporção de 2:2:1 volume/volume. $\mathrm{O}$ inoculante comercial foi obtido com material base de semente de trigo esterilizada e colonizada com o fungo $P$. ostreatus (shimeji branco). 


\section{Montagem do experimento}

O experimento foi montado de acordo com a Figura 1, em que os materiais anteriormente citados foram misturados e amontoados em medas de compostagem de $1 \mathrm{~m}^{3}$, sendo revirados a cada dois dias por um período de uma semana e, após, condicionados em túnel com injeção de vapor em fluxo contínuo durante 12 horas (SIQUEIRA et al., 2012). O material de cada parcela foi inserido em sacos transparentes com capacidade de $4 \mathrm{Kg}$.

O composto presente em cada saco foi inoculado com: 1) $200 \mathrm{~g}$ de inoculante comercial ( $5 \%$ em relação ao peso do composto) ou 2) alíquota de $600 \mathrm{~g}$ de composto (matriz) previamente colonizado por micélio de $P$. ostreatus (shimeji branco) (15\% em relação do peso do composto), caracterizando os tratamentos experimentais. As parcelas experimentais foram replicadas em quatro repetições e distribuídas em três andares de prateleiras. Esse procedimento foi repetido após sete dias, visando minimizar as fontes de oscilação experimentais (compostagens 1 e 2).

\section{Avaliação do crescimento micelial}

Para o acompanhamento do desenvolvimento micelial (mm.dia $\left.{ }^{-1}\right)$, foram utilizados sacos cilíndricos transparentes com $1 \mathrm{Kg}$ de composto e inoculados somente em sua superfície superior. A avaliação iniciou após sete dias da inoculação dos sacos, por meio da delimitação da divisa entre o material colonizado e o não colonizado, com uso de caneta marcadora permanente e, após sete dias, foi realizada nova marcação. $O$ intervalo entre as delimitações permitiu demarcar seu desenvolvimento micelial e, com auxílio de uma trena, medir, em cinco pontos equidistantes, o desenvolvimento do micélio, e calcular a média dos pontos, dividindo pelo número de dias, entre as duas demarcações, assim obtendo a média de crescimento diário de cada parcela experimental.

\section{Colheita e cálculo de produtividade}

Após o tempo de incubação e completa colonização micelial no composto, as parcelas foram acondicionadas na casa de cultivo, em prateleiras, por sorteio aleatório. Para iniciar a frutificação, toda superfície lateral e superior dos compostos de cultivo foi furada, respeitando uma distância de $10 \mathrm{~cm}$ de cada furo, ocasionando maior oferta de oxigênio no substrato $e$, consequentemente, o desenvolvimento dos cogumelos. A colheita foi realizada três vezes ao dia, para a padronização do tamanho dos cogumelos, que foram pesados e anotados em planilhas, permitindo o cálculo futuro da produtividade após 84 dias (setembro-dezembro) de colheita. Para este cálculo, foi considerada a massa de cogumelos frescos colhidos, dividida pela massa de composto úmido, em porcentagem $[\mathrm{P}=$ (massa de cogumelos frescos/massa de composto úmido) x 100].

\section{Análise estatística}

A análise estatística foi realizada com o auxílio do software Sisvar ${ }^{\circledR}$ (FERREIRA, 2011). O delineamento do experimento foi em blocos casualizados, sendo considerados os níveis das prateleiras como variáveis ambientais, e as diferentes formas de inoculação como fontes de variação.

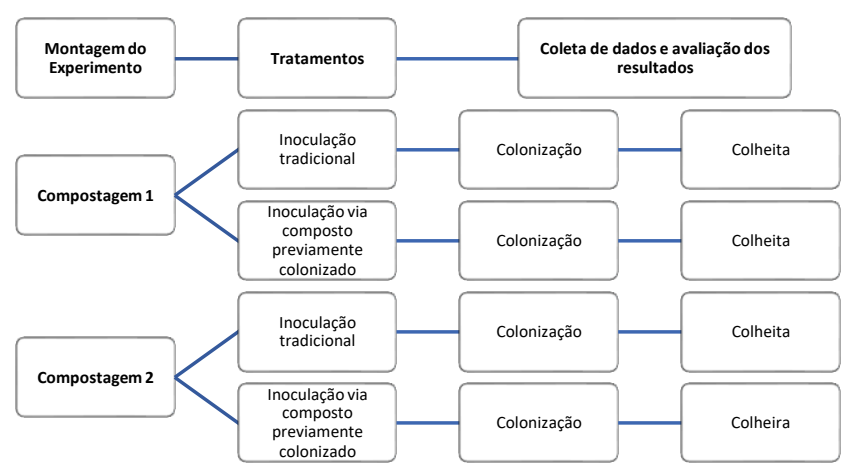

Figura 1. Desenvolvimento do experimento, desde a montagem da compostagem, inoculação, colonização e colheita dos cogumelos.

\section{RESULTADOS E DISCUSSÃO}

O experimento comparativo dos dois métodos de inoculação do composto ("spawn" versus alíquota de composto previamente colonizado) foi monitorado em estufa, sob condições controladas de cultivo. Para o crescimento micelial, não foi observada diferença estatística entre os compostos que foram inoculados com "spawn" comercial (média de 5,27 mm.dia-1) e com composto previamente colonizado $\left(5,17 \mathrm{~mm}^{-d_{i a}{ }^{-1}}\right.$ ) (Tabela 1). Isso sugere a boa capacidade de colonização dos micélios coletados previamente de composto colonizado.

Após o período de incubação e, portanto, aos primeiros sinais de colonização de toda a superfície do composto, deu-se início à coleta dos corpos de frutificação para pesagem e anotação em planilha. A quantificação de biomassa de cogumelos produzida por cada parcela de composto permitiu comparar as diferentes formas de inoculação, bem como eliminar qualquer dúvida em 
relação aos resultados por meio das réplicas do processo de compostagem, visto que o coeficiente de variação é alto em experimentos com cultivos de cogumelos comestíveis, induzindo a dúvidas em relação aos dados obtidos (DIAS, 2010; SIQUEIRA et al., 2011).

Tabela 1. Média de crescimento micelial, em milímetros por dia, comparando as duas metodologias de inoculação do composto.

Tratamento

\begin{tabular}{cl}
\hline Spawn & $5,27 \mathrm{~A}^{*}$ \\
Composto colonizado & $5,17 \mathrm{~A}$ \\
\hline
\end{tabular}

*As médias seguidas de mesma letra não diferem estatisticamente, de acordo com o teste de Scott-Knott, em nível de 5\% de probabilidade.

Para a primeira compostagem, a produtividade dos dois tratamentos foi estatisticamente igual, em nível de $5 \%$ de certeza, de acordo com o teste de Scott-Knott; os compostos de cultivos inoculados utilizando a metodologia tradicional atingiram produtividade média de $17,54 \%$, e as parcelas inoculadas a partir de compostos previamente colonizados atingiram 17,64\% (Tabela 2), com média de 701,42 e 705,75 gramas, respectivamente (Figura 2).

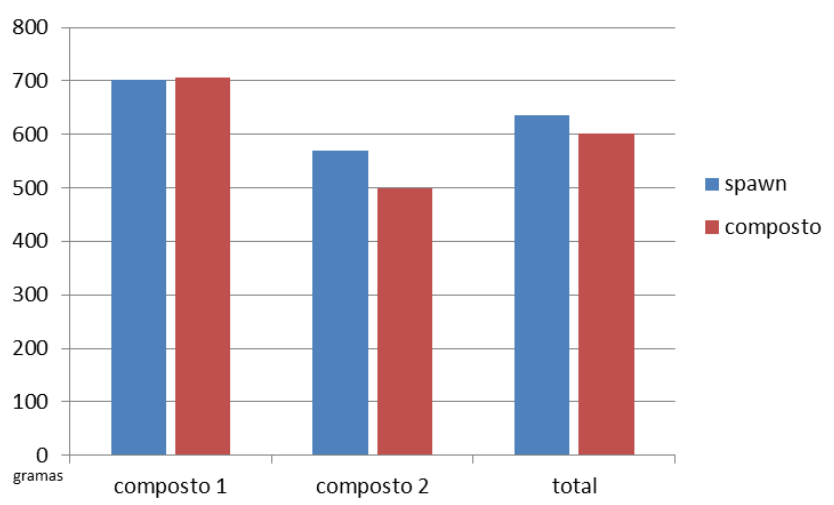

Figura 2. Produção, em gramas, de cogumelo $P$. ostreatus para os diferentes tratamentos de inoculação e diferentes experimentos de compostagem.

Para a segunda compostagem, os compostos de cultivos inoculados utilizando a metodologia tradicional atingiram produtividade média de $14,22 \%$, e as parcelas inoculadas a partir de compostos previamente colonizados atingiram 12,47\% (Tabela 2), com média de 568,83 e 498,92 gramas, respectivamente (Figura 2), da mesma forma igual, estatisticamente. Quando comparamos os mesmos tratamentos com as duas compostagens, percebemos estatisticamente uma produtividade inferior na segunda compostagem, para ambos os tratamentos
(Tabela 2).

Tabela 2. Média de produtividade, em percentual, comparando as diferentes metodologias de inoculação e diferentes compostos; e média de produtividade dos dois compostos.

\begin{tabular}{cccc}
\hline \multirow{2}{*}{ Tratamento } & \multicolumn{3}{c}{ Produtividade (\%) } \\
\cline { 2 - 4 } & Composto 1 & Composto 2 & Média \\
\hline Spawn & $17,54 \mathrm{Aa}^{*}$ & $14,22 \mathrm{Ab}$ & 15,88 \\
$\begin{array}{c}\text { Composto } \\
\text { colonizado }\end{array}$ & $17,64 \mathrm{Aa}$ & $12,47 \mathrm{Ab}$ & 15,05 \\
\hline $\begin{array}{l}\text { *As médias seguidas de mesma letra maiúscula na coluna e minúscula } \\
\text { na linha não diferem estatisticamente, de acordo com o teste de Scott- } \\
\text { Knott, em nível de 5\% de probabilidade. }\end{array}$ &
\end{tabular}

O experimento comparativo de dois diferentes métodos de inoculação de composto - via semente comercial e alíquota de composto colonizado - permitiu inferir que não houve diferença, na produtividade, que pudesse ser ocasionada pela inoculação a partir de composto colonizado. A repetição do experimento permitiu inferir que qualquer variação na qualidade, metodologia de compostagem e variação ambiental, sobre algumas das quais o produtor não possui controle total, podem influenciar na produtividade dos cogumelos.

Em contrapartida, os sacos de compostos inoculados com composto colonizado apresentaram um desenvolvimento micelial menos vigoroso, ou seja, visualmente menos denso. Isso demonstra a necessidade de cautela ao afirmar que essa metodologia poderia ser repetida por vários ciclos, pelo risco da perda da vitalidade do fungo e, também, pelo risco de contaminação por micro-organismos oportunistas, quando comparado aos compostos inoculados com a técnica tradicional ("spawn").

Em virtude dessa observação, é importante ressaltar a necessidade de seleção do composto colonizado que servirá de matriz para a inoculação; este deve ser sadio e vigoroso, isto é, livre de contaminantes e pragas, tampouco deve ter iniciado a sua produção de cogumelos, visto que o corpo de frutificação drena nutrientes com maior potencial do substrato, em comparação ao micélio em desenvolvimento.

Assim, essa técnica pode ser considerada uma alternativa viável em situações em que o produtor não tenha a pronta disponibilidade de inóculos de qualidade. As principais precauções tomadas nesse experimento foram o delineamento em diferentes níveis de prateleira, que permitiu excluir qualquer variação ambiental que pudesse influenciar na produtividade, diminuindo erros 
estatísticos da avaliação na produção de corpos de frutificação nas parcelas experimentais. Essas diferenças poderiam ser ocasionadas pela variação da oferta de aeração e umidade ao longo da estufa de cultivo, bem como diferenças de altura das prateleiras.

As réplicas de compostagem que foram produzidas em um intervalo distante de sete dias já apresentaram diferenças na produção, podendo ter sido ocasionada tanto por variações climáticas quanto diferenças na qualidade do material utilizado. Comercialmente, formulações com base em grãos de trigo são mais comumente utilizadas como substrato de crescimento de micélios de cogumelos comestíveis entre os produtores de "spawn", uma vez que proporciona rápida multiplicação celular proporcionada pelo estoque energético das sementes e, por conseguinte, excelente desempenho na colonização do substrato, e posterior produção de corpos de frutificação (ROSADO et al., 2002). Por outro lado, seu uso em larga escala é limitado a pequenos fornecedores, devido ao alto custo e probabilidade de contaminação, fazendo com que grandes fornecedores, cooperativas e instituições públicas busquem alternativas para produção de inóculo a custo reduzido e por meio de tecnologias laboratoriais, como é o caso de culturas líquidas e suas adaptações (LIU et al., 2018; ZHANG et al., 2019).

Os resultados do experimento também oportunizam o uso de inóculo de procedência sustentável e agroecológica, visto que haja interesse pela aquisição de hortaliças frescas no Brasil, cujo processo produtivo não tenha empregado substratos ou insumos químicos que comprometam o equilíbrio dos ecossistemas, ou que seja possível de atingir um percentual de $95 \%$ de componentes de origem orgânica ao longo da escala produtiva (MAPA, 2016). O cultivo de cogumelos comestíveis não deve empregar aditivos químicos que tenham efeito biocida sobre pragas, em virtude da capacidade desses fungos comestíveis bioacumularem esses compostos durante a formação dos corpos de frutificação (CARVALHO et al., 2014).

Ainda, o cultivo de cogumelos por olericultores é válido, uma vez que oportuniza a ciclagem de nutrientes gerados na propriedade, de forma que o resíduo agrícola possa ser utilizado como substrato de cultivo de cogumelos (MANDEEL et al., 2005; SIQUEIRA et al., 2011; SINGH e SINGH, 2012; SIQUEIRA et al., 2012) e, posteriormente, o composto exaurido, obtido ao final dos ciclos de frutificação dos cogumelos, possa ser utilizado como substrato para a produção de mudas de hortaliças e/ou aplicado em canteiros de culturas de hortaliças como condicionador de solo, como demonstrado na Figura 3, oportunizando o retorno de nutrientes e dando uma significação agroecológica e sustentável a sua produção, agregando valor à atividade econômica do pequeno produtor rural (MARQUES et al., 2014; LOPES et al., 2015; BELLETTINI et al., 2017).

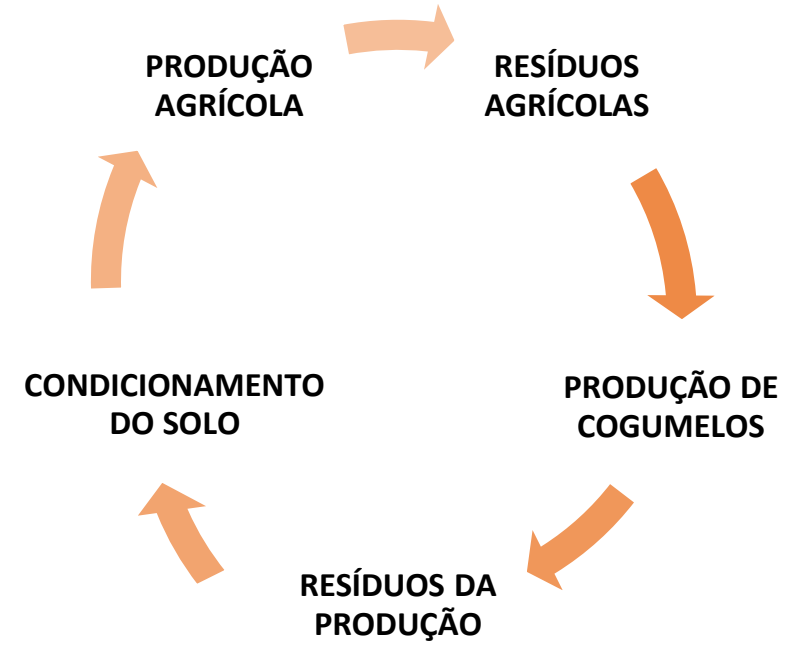

Figura 3. Fluxograma da ciclagem de nutrientes oportunizada pela produção agrícola contemplada com o cultivo de cogumelos comestíveis.

Agregando valor a esse fato, o cultivo de cogumelos oportuniza, ao agricultor, um substrato agrícola de qualidade e orgânico, permitindo sua aplicação em culturas certificadas perante o Ministério da Agricultura, Pecuária e Abastecimento (MAPA), além de ser um importante aporte de renda; mesmo produtores de hortaliças que utilizam a metodologia convencional de cultivo podem utilizar os resíduos da produção de cogumelos, como forma de redução de custos (MARQUES et al., 2014; LOPES et al., 2015), visto que técnicas agroecológicas de manejo e adubação melhoram o equilíbrio ecológico do solo, permitindo uma melhor ciclagem e aproveitamento de nutrientes (LEIJSTER et al., 2019).

\section{CONCLUSÕES}

A inoculação de substrato de cultivo do cogumelo $P$. ostreatus a partir de alíquota de composto previamente colonizado por micélio é viável em ocasiões de indisponibilidade de inoculantes, sendo uma técnica sustentável. Mais experimentos devem ser conduzidos a fim de avaliar essa produtividade com outras linhagens de $P$. ostreatus, bem como com outros gêneros de cogumelos comestíveis, com o objetivo de concluir a viabilidade na 
substituição do inoculante comercial e/ou o número de vezes que essa metodologia possa ser repetida, facilitando a disseminação de metodologias de emergência e favoráveis às condições agroecológicas e do pequeno produtor brasileiro.

\section{REFERÊNCIAS}

ANPC - ASSOCIAÇÃO NACIONAL DE PRODUTORES DE COGUMELOS. Cogumelos no Brasil. Pleurotus spp. - Cogumelo Ostra. 2018. Disponível em: https://www.anpccogumelos.org/

BELLETTINI, M.B. et al. Residual compost from the production of Bactris gasipaes Kunth and Pleurotus ostreatus as soil conditioners for Lactuca sativa 'Veronica'. Semina: Ciências Agrárias, v.38, n.2, p.581-593, 2017.

CARVALHO, C.S.M. et al. Applicability of the use of waste from different banana cultivars for the cultivation of the oyster mushroom. Brazilian Journal of Microbiology, v.43, n.2, p.819826, 2012

CARVALHO, M.A. et al. Bioaccumulation of insecticide in Agaricus subrufescens. Horticultura Brasileira, v.32, p.159-162, 2014.

CHITAMBA, J. et al. Evaluation of substrate productivity and market quality of oyster mushroom (Pleurotus ostreatus) grown on different substrates. International Journal of Agricultural Research, v.7, n.2, p.100-106, 2012.

CLIMATEMPO. Climatologia Bom Sucesso-MG. 2018. Disponível em: https://www.climatempo.com.br/climatologia/3614/bom sucesso-mg

CONTRERAS, E.P. et al. Soaking of substrate in alkaline water as a pretreatment for the cultivation of Pleurotus ostreatus. The Journal of Horticultural Science and Biotechnology, v.79, n.2, p.234-240, 2004.

DIAS, E.S. Mushroom cultivation in Brazil: challenges and potential for growth. Ciência e Agrotecnologia, v.34, n.4, p.795803, 2010.

FERREIRA, D.F. Sisvar: a computer statistical analysis system. Ciência e Agrotecnologia, v.35, n.6, p.1039-1042, 2011.

GIRMAY, Z. et al. Growth and yield performance of Pleurotus ostreatus (Jacq. Fr.) Kumm (oyster mushroom) on different substrates. AMB Express, v.6, n.87, p.1-17, 2016.

LEIJSTERA, V. et al. Agroecological management improves ecosystem services in almond orchards within one year. Ecosystem Services, v.38, p.1-12, 2019.

LIU, S.R. et al. Production of stalk spawn of an edible mushroom (Pleurotus ostreatus) in liquid culture as a suitable substitute for stick spawn in mushroom cultivation. Scientia Horticulturae, v.240, p.572-577, 2018.

LOPES, R.X. et al. Application of spent Agaricus subrufescens compost in integrated production of seedlings and plants of tomato. International Journal of Recycling of Organic Waste in
Agriculture, v.1, p.1-8, 2015.

MANDEEL, Q.A. et al. Cultivation of oyster mushrooms (Pleurotus spp.) on various lignocellulosic wastes. World Journal of Microbiology \& Biotechnology, v.21, p.601-607, 2005.

MANTOVANI, T.R.D. et al. Formulação de substratos na produção de biomassa micelial e de lacase de Pleurotus ostreatus. Semina: Ciências Agrárias, v.33, n.5, p.1681-1692, 2012.

MAPA - MINISTÉRIO DA AGRICULTURA, PECUÁRIA E ABASTECIMENTO. O que são produtos orgânicos? 2016. Disponível em: http://www.agricultura.gov.br/assuntos/ sustentabilidade/organicos

MARQUES, E.L.S. et al. Spent mushroom compost as a substrate for the production of lettuce seedlings. Journal of Agricultural Science, v.6, p.138-143, 2014.

OBODAl, M. et al. Comparative study on the growth and yield of Pleurotus ostreatus mushroom on different lignocellulosic byproducts. Journal of Industrial Microbiology \& Biotechnology, v.30, p.146-149, 2003.

OGIDI, O.C. et al. Mycelial growth, biomass production and iron uptake by mushrooms of Pleurotus species cultivated on Urochloa decumbens (Stapf) R. D. Webster. Journal of Food Research, v.5, n.3, p.13-19, 2016.

RIBAS, L.C.C. et al. Use of spent mushroom substrates from Agaricus subrufescens (syn. A. blazei, A. brasiliensis) and Lentinula edodes productions in the enrichment of a soil-based potting media for lettuce (Lactuca sativa) cultivation: Growth promotion and soil bioremediation. Bioresource Technology, v.100, p.47504757, 2009.

ROSADO, F.R. et al. Alternative method of inoculum and spawn production for the cultivation of the edible Brazilian mushroom Pleurotus ostreatoroseus SING. Journal of Basic Microbiology, v.42, n.1, p.37-44, 2002.

SALES-CAMPOS, C. et al. Cinética micelial dos fungos comestíveis Pleurotus ostreatus e Lentinula edodes em resíduos lignocelulósicos. Arquivos do Instituto Biológico, v.78, n.1, p.141145, 2011a.

SALES-CAMPOS, C. et al. Physiochemical analysis and centesimal composition of Pleurotus ostreatus mushroom grown in residues from the Amazon. Ciência e Tecnologia de Alimentos, v.31, n.2, p.456-461, 2011b.

SÁNCHEZ, C. Cultivation of Pleurotus ostreatus and other edible mushroom. Applied Microbiology and Biotechnology, v.80, n.5, p.1321-1337, 2010.

SILVA, M.C.S. et al. Mycelial growth of Pleurotus Spp in Seenriched culture media. Advances in Microbiology, v.3, p.11-18, 2013.

SINGH, M.P.; SINGH, V.K. Biodegradation of vegetable and agrowastes by Pleurotus sapidus: a novel strategy to produce mushroom with enhanced yield and nutrition. Cellular \& Molecular Biology, v.58, p.1-7, 2012. 
SIQUEIRA, F.G. et al. Cultivation of Pleurotus sajor-caju cultivation on banana stalk and Bahia grass based substrates. Horticultura Brasileira (Impresso), v.29, p.199-204, 2011.

SIQUEIRA, F.G. et al. Cultivation of Pleurotus mushrooms in substrates obtained by short composting and steam pasteurization. African Journal of Biotechnology, v.11, n.53, p.11630-11635, 2012.

VIEIRA, F.R.; ANDRADE, M.C.N. Optimization of substrate preparation for oyster mushroom (Pleurotus ostreatus) cultivation by studying different raw materials and substrate preparation conditions (composting: phases I and II). World Journal of Microbiology and Biotechnology, v.32, n.11, p.190, 2016.

ZADRAZIL, F. Influence of CO2 concentration on the mycelium growth of three Pleurotus species. European Journal of Applied Microbiology, v.1, p.327-225, 1975.

ZHARARE, G.E. et al. Effects of temperature and hydrogen peroxide on mycelial growth of eight Pleurotus strains. Scientia Horticulturae, v.125, p.95-102, 2010.

ZHANG, W.R. et al. Development of a novel spawn (Block Spawn) of an edible mushroom, Pleurotus ostreatus, in liquid culture and its cultivation evaluation. Mycobiology, v.47, n.1, p.97-104, 2019. 